\title{
Cortisol Awakening Response: An Ancient Adaptive Feature
}

\section{Carlos M. Contreras ${ }^{1,2 *}$ and Ana G. Gutiérrez-Garcia ${ }^{2}$}

${ }^{1}$ Unidad Periférica Xalapa, Instituto de Investigaciones Biomédicas, Universidad Nacional Autónoma de México, Xalapa, Veracruz, 91190, México

${ }^{2}$ Laboratorio de Neurofarmacología, Instituto de Neuroetología, Universidad Veracruzana, Xalapa, Veracruz, 91190, México

"Corresponding Author: Dr. Carlos M. Contreras, Dr. Sci., Laboratorio de Neurofarmacología, Av. Dr. Luis Castelazo s/n, Col. Industrial Ánimas, Xalapa, Veracruz, 91190, México, Tel: +52 (228) 8418900, Ext: 13613; Fax: +52 (228) 8418918; E-mail: ccontreras@uv.mx (or) contreras@ biomedicas.unam.mx

Received: 31 January 2018; Accepted: 20 February 2018; Published: 27 February 2018

\begin{abstract}
Similar to other endocrine substances, cortisol secretion follows a pulsating rhythm. The cortisol awakening response (CAR) occurs upon awakening in the absence of any apparent stressful situation or imminent danger, which is a very intriguing feature. When confronting any stressful situation, two systems are activated. One system is regulated by the hypothalamic-pituitary-adrenal axis (HPA), and the other system is regulated by cerebral structures that control the activity of the autonomic sympathetic nervous system. Both systems receive inputs from emotional memory circuits, namely the amygdala, the hippocampus, the medial prefrontal cortex, and lateral septal nuclei, among others. This circuit integrates sensory information that comes from thalamic nuclei. The acquisition, retention, and evocation of recent and remote memories that are processed by the emotional memory circuit allow the selection of strategies for survival. The diurnal secretion of cortisol occurs near the time of awakening (i.e., after a period of rest or sleeping) and persists for several hours in the absence of any current stressful situation. The CAR seems to represent an ancient adaptive-allostatic feature that prepares an individual to face eventualities that are forthcoming during the day. The CAR is regulated by hypothalamic nuclei that modulate circadian rhythm, namely the suprachiasmatic nucleus and its connections with the paraventricular nucleus, and then activate the HPA axis. The CAR may represent a useful preparatory process that occurs before a stressful situation. The participation of emotional memory circuits may modify the CAR and contribute to resilient or vulnerable reactions when coping with threatening situations.
\end{abstract}


Keywords: Adaptive; Allostasis; Allostatic load; Ancient; Anxiety; Cortisol; Cortisol awakening response; Stress

\section{Introduction}

Throughout the day, plasma cortisol levels typically peak many times. A period of low plasma concentrations generally centers around midnight, with an abrupt rise that commonly occurs after awakening, independent of age, gender, and other aspects [1]. The cortisol awakening response (CAR) is an indicator of adrenocortical activity that consists of an increase in plasma cortisol within the first hour after waking. Within the first 30-40 min after awakening, free cortisol levels rise by 50-60\%, remain elevated for at least $60 \mathrm{~min}$ [2], and decline to a nadir thereafter by about bedtime.

An approach to understanding the processes that allow individuals to adapt to their environment is called allostasis $[3,4]$. This concept refers to functional changes in hormones and mediators that occur in an organism that allow the individual to confront perturbations in the internal and external milieus. These changes permit the survival of the individual and consequently the species. Allostasis depends on the activity of two main systems: (i) hypothalamicpituitary-adrenal (HPA) axis and (ii) autonomic nervous system. During the day, cortisol levels may increase in response to emergencies, whereas the CAR may be an anticipatory response that is directed toward daily eventualities just after awakening. However, in cases in which high levels of cortisol persist for a long period of time, are inefficiently managed, or become exaggerated, allostatic load may occur [4-6], which can negatively impact health.

The organism is able to respond to emergency situations through physiological adaptive changes that permit the individual to maintain homeostasis and survive. From a psychological perspective, this response is referred to as resilience, which reflects the ability of the living organism to face and overcome stressful situations [7]. In other cases, some maladaptive processes may be related to vulnerability [8-10]. However, HPA and autonomic activity is insufficient to explain resilience and vulnerability. Increases in cortisol when confronting an emergency situation and the CAR may prepare the individual for future emotional threatening events, thus suggesting the participation of brain circuits that are involved in emotional processing.

The present review considers the participation of emotional memory circuits in the regulation of endocrine and autonomic responses both at rest and when confronting a threatening situation. Cortisol has been considered a marker of stress [11], in addition to other products of autonomic nervous system activity. Acute stressors activate the HPA axis, leading to the release of corticotropin-releasing factor into the portal circulation (Figure 1). Adrenocorticotropic hormone ( $\mathrm{ACTH})$ is then released into the plasma, and the cortical portion of the adrenal gland is activated to deliver cortisol into the circulation. Plasma cortisol levels reach a peak approximately 15-30 min after an environmental challenge [12]. 


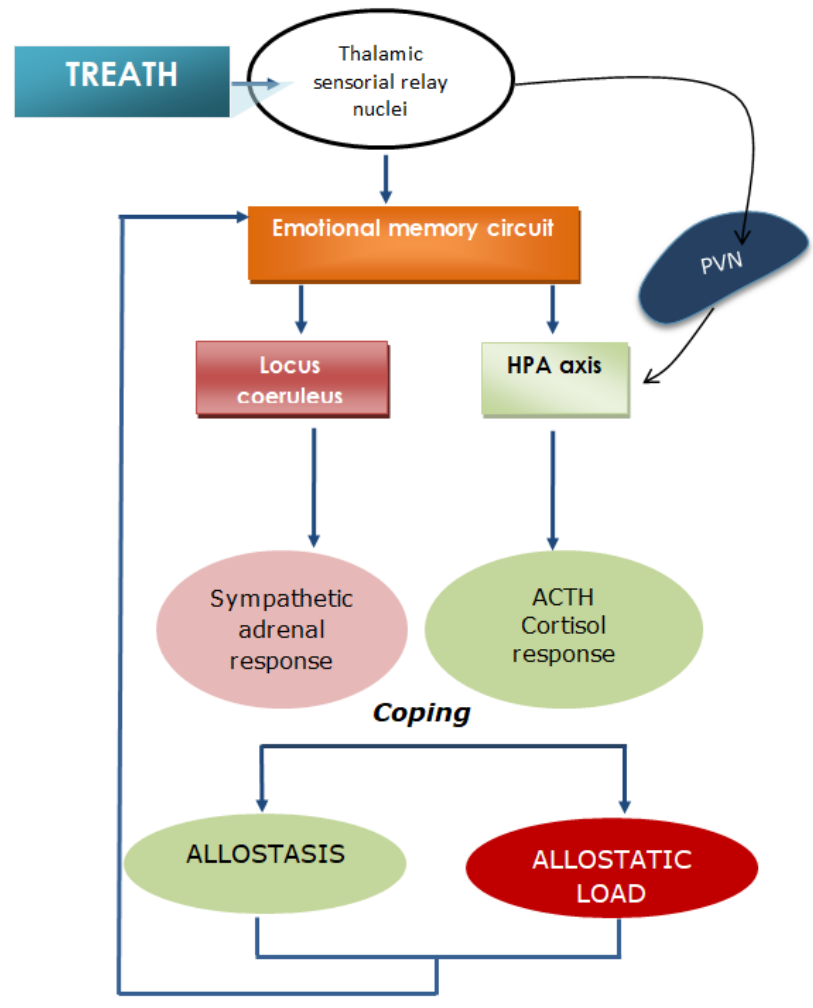

Figure 1: A threatening situation elicits two simultaneous responses: sympathetic responses and cortisol secretion that is regulated by the HPA axis. These two systems interact with each other. The participation of cerebral nuclei that regulate emotional memory processing may explain susceptibility and resilience to stress. ACTH, adrenocorticotropic hormone; HPA, hypothalamic-pituitary-adrenal axis; PVN, paraventricular nucleus of the hypothalamus; mPFC, medial prefrontal cortex.

The cortisol response when confronting stressful situations has been extensively reviewed elsewhere; therefore, we only briefly discuss it herein. We focus mainly on the CAR, beginning with a brief overview of the brain structures that regulate emotional memory and its relationships with brain structures that regulate cortisol secretion. We then discuss the specific features of the CAR, its neural control, and the cortisol response to cope with threatening situations in other vertebrates. The hypothesis of the present treatise is that the CAR may represent a very useful ancient adaptive response.

\section{Emotional Memory Circuit}

Emotional memory allows an individual to recognize signs from the environment and compare them with past experiences to effectively judge and respond to the environment by choosing the best coping strategy [13, 14]. Such processes involve the hippocampus and other deep temporal lobe structures, such as the amygdala [15], the mesolimbic system [16], and interactions among these structures and the prefrontal cortex [17, 18], among other connections. Sensory inputs relies on thalamic nuclei that are connected to cortical and subcortical cerebral circuits [19] that regulate the emotional meaning of stimuli and endocrine and autonomic responses. 
The neural circuits that regulate emotional memory comprise several interrelated structures that are located primarily in deep layers of the temporal and frontal lobes that project to the HPA axis and cerebral regulators of corticosterone secretion and adrenergic system activation (Figure 2).

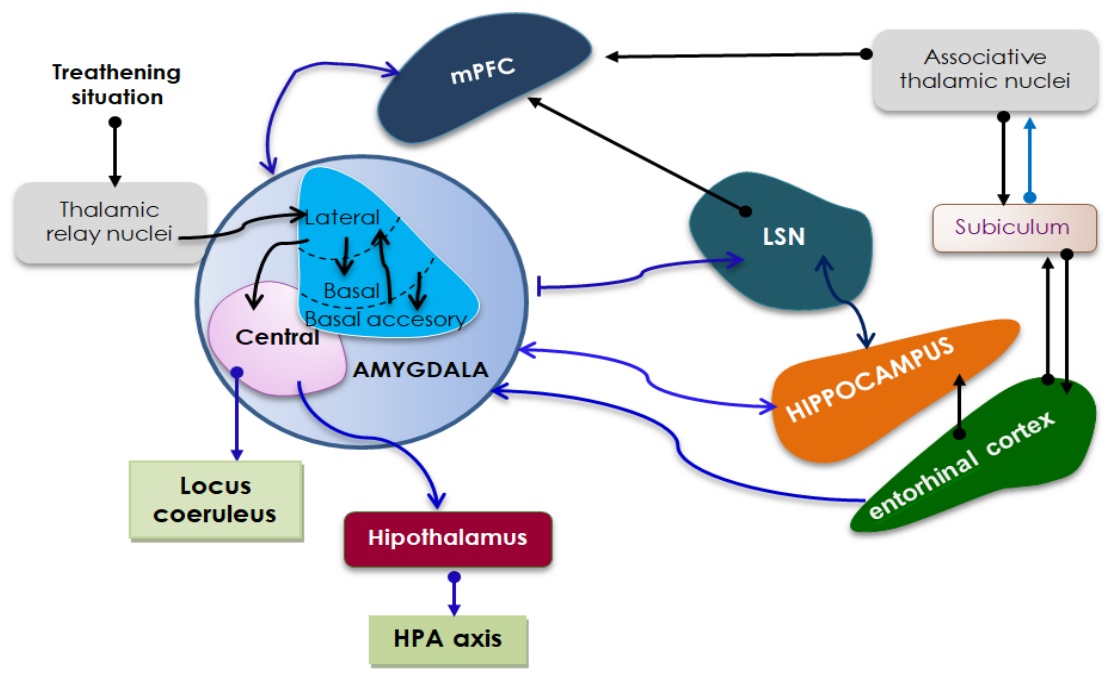

Figure 2: Anatomical representation of emotional memory circuit. Connections between the amygdala, hippocampus, lateral septal nucleus (LSN), and medial prefrontal cortex (mPFC) modulate the utilization of emotional memories. These nuclei are also connected to the locus coeruleus and hypothalamus, which are involved in autonomic responses and cortisol secretion.

Among other temporal lobe structures, the amygdala complex is composed of many functionally heterogeneous nuclei [20]. The amygdala nuclei have been largely considered as fundamental in the process and integration of defensive and fear reactions [21-23]. Basolateral amygdala includes basal, lateral and accessory basal nuclei [24] and fear reactions [25], increased anxiety state [26], during the processes of emotional learning [27] and classic conditioning [28] relates to a higher neuronal firing rate in these regions than in absence of stimulation or resting situations. From a behavioral point of view, electrical stimulation of amygdala produces signs of fear and anxiety, accompanied by vegetative responses in both cats [29] and human beings [30]. Fear expression involves cortical association areas, and thalamic and amygdaline interconnections [31]; importantly, cortisol seems to regulate the connectivity between amygdala and at least the medial prefrontal cortex (mPFC) inclusively during rest conditions [32], while amygdala-hypothalamic connections regulate vegetative activity in response to threatening situations [33].

Among another amygdaline connections, the reciprocal innervation with hippocampus modulate the unconditioned fear, defense reactions, goal-directed behavior and emotional memory [34, 35], with the important participation of the two different portions of hippocampus [36]. Therefore, amygdala-hippocampus relations are crucial in the control and regulation of episodic memory and emotional memory, and as above mentioned, through the connections of amygdala with hypothalamus in the control of cortisol secretion. In rats, the corresponding portions are the dorsal and ventral hippocampus, which are related to memory and emotional processing, respectively [37]. The responsivity of dorsal hippocampal neurons responders to amygdala stimulation increased $48 \mathrm{~h}$ after a single session of stress, suggesting the formation of an emotional memory [38]. Increases in endogenous cortisol and 
norepinephrine levels in turn increase neuronal activation in the amygdala in response to threatening images [39]. In such cases, the higher levels of plasma cortisol when confronting a threatening situation may facilitate specific learning that is relevant to survival [40].

Amygdala-mPFC connections are able to regulate aggressive behavior in rodents [41-43]. In rats, the mPFC involves the cingulate, prelimbic (PL) and infralimbic (IL) subregions, each subregion possess different connections and consequently different functions. In particular PL and IL differentially regulate the expression of fear [44], among other behaviors [17], possibly due to their interconnections with amygdala [45]. mPFC subregions differentially participate in the process of acquisition and extinction of conditioned fear [46, 47] through inhibitory connections coming from amygdala [28], thus mediating distinct strategies to cope with environment. Inactivation of the PL cortex impaired the expression of fear but not extinction memory. Inactivation of the IL cortex had no effect of the expression of fear but impaired both the acquisition and extinction of conditioned fear memories [46]. Activation of the PL and IL regions has yielded consistent results. The PL cortex is active during fear conditioning, and the IL cortex becomes active during fear extinction [47].

Another structure that is connected to the amygdala, hippocampus, and mPFC is the lateral septal nucleus. Together with the aforementioned key regions, the lateral septal nucleus also participates in the control of motivational and autonomic responses [48], the antidepressant actions of drugs [49], anxiety [50], affective behavior, and autonomic activity [51].

Brain structures that are related to emotional memory appear to influence and may be influenced by the actions of cortisol secretion and sympathetic activity. In such a case, the participation of emotional memory circuits due to its function of retention of experiences related with threatening situations may account for the formation of resilience and vulnerability, and consequently modifying the vegetative responses, favoring or negatively impacting on the efficacy of allostatic processes.

\subsection{Cortisol awakening response and sleep}

The diurnal increase in cortisol secretion is associated with the sleep/wake and light/dark cycles. The CAR is a very constant feature that is modulated by circadian influences. In very young children, the level of morning cortisol is positively associated with the amount of stage-2 sleep the night before and negatively associated with total sleep time and other slow-wave-sleep stages [52].

Total sleep deprivation in healthy adults decreases the CAR in parallel with changes in the perception of energy level, concentration, and speed of thought and a reduction of cognitive functioning despite an increase in regional dopaminergic activity [53]. Chronic circadian misalignment significantly reduced cortisol levels and increased the release of inflammatory factors, including tumor necrosis factor, interleukin, and C-reactive protein [54]. The interaction between sleep and the HPA axis is complex and bidirectional. Hypothalamic-pituitary-adrenal axis hyperactivity and decreases in the duration and quality of sleep occur in insomnia, depression, Cushing's syndrome, and sleep-disordered breathing, among other ailments [55]. Changes in sleep duration contribute to daily variations in cortisol and autonomic nervous system activity [56].

J Psychiatry Psychiatric Disord 2018; 2 (1): 29-40 


\subsection{Neural regulation of the cortisol awakening response}

The suprachiasmatic nucleus regulates the circadian rise in plasma ACTH [57,58]. Suprachiasmatic nucleus regulates activity on paraventricular hypothalamic nucleus and exerts a decisive action on the day/night pattern of hormonal and autonomic activity regulation [59]. This anatomical feature regulates CAR and the influence of ACTH on suprarenal cortex [60].

Sensorial stimulation produces emotional reactions and elaborated behaviors (Figure 3). The hypothalamic regulation CAR [61] is modulated by a multiple system of neurotransmission, mainly glutamatergic, aspartate, and GABAergic fibers from telencephalic and forebrain regions, which are considered limbic structures [62-64], but not from the lower brainstem. These hypothalamic nuclei control the neuroendocrine response to stress, whereas the extended amygdala controls the autonomic responses to stress [12]. Therefore, the paraventricular nucleus may be considered an integrator of neuroendocrine and autonomic nervous system responses and may also participate in the integrated emotional response. The CAR may also be involved in the activation of a negative feedback loop that results in the termination of ACTH secretion [65]. Anxiety may be a useful adaptive feature [14] that, combined with the storage of emotional memories of prior experiences, facilitates the choice of the best strategies for survival.

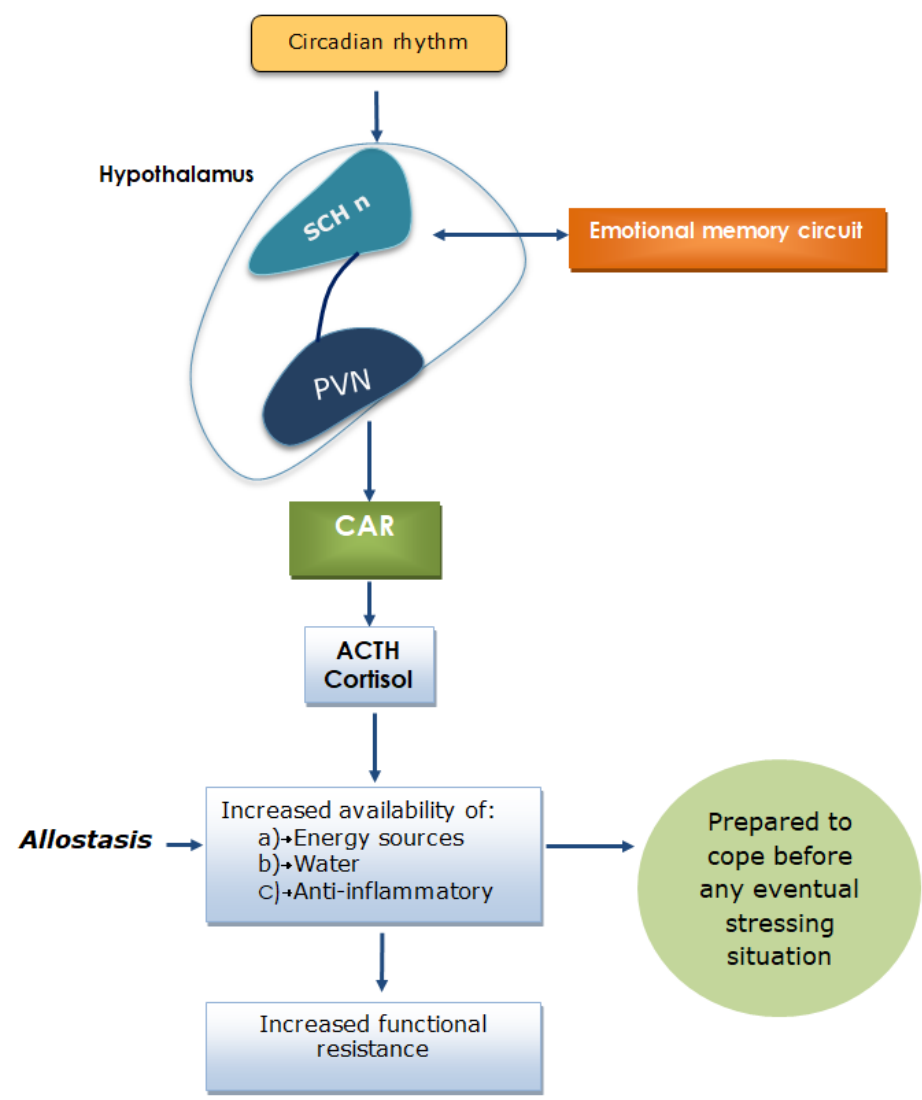

Figure 3: The circadian rhythm of cortisol release occurs in the absence of a threatening situation. Therefore, it may be considered a useful allostatic adaptive feature that prepares an individual for eventual emergency situations, with

the fundamental participation of emotional memory circuits. PVN, paraventricular nucleus of the hypothalamus;

$\mathrm{SCH}$ n: suprachiasmatic nucleus; CAR: cortisol awakening response. 


\subsection{Cortisol in other animal species}

The cortisol response to threatening situations is not exclusive to humans or other mammals. Individuals that present similar HPA axis function express similar responses to threatening situations, independent of species. A rise in cortisol may indicate the development of behavioral strategies that facilitate escape from predators and functional metabolic changes that allow survival through allostasis.

In the presence of predators or threatening situations, cortisol (or corticosterone) is released by fish [66-69], amphibians [70], small mammals [71-74], goats [75], and seals [76]. This rise in cortisol (or corticosterone) allows suppressive behavioral actions (e.g., freezing) in some cases and preparative defensive actions (e.g., attack) in others $[77,78]$. For example, increases in cortisol may mobilize glucose for sustained vigilance and running during periods of reduced foraging possibilities [78]. It is currently unknown whether such increases in circulating cortisol in other vertebrates follow a circadian rhythm or occur after periods of sleep or rest. The delivery of cortisol by the adrenal glands and other metabolic processes may be related to a functional preparatory reaction of the organism to a threating situation that allows individuals to adapt to their environment.

\section{Conclusion}

The processes that are involved in the sequence of events that allows us to cope with stress appear to represent an adaptive process that slowly developed in our ancestral past [79]. The increase in glucocorticoid levels upon awakening prepares the body for activity, thus enabling foraging behavior by increasing the amount of energy that is available $[60,80]$. Homo sapiens have not appreciably changed for a long time. As a species, we are exactly alike. One function of the CAR may be to energize people in the morning [81].

Early in the morning, a relatively high amount of cortisol is released, and cortisol levels dramatically increase after a few minutes, leading to exploratory behavior, food seeking, and the facilitation of typical behavioral patterns of each species to survive [82]. Upon awakening, our body is ready to hunt and fight, being previously prepared to support thirst and hunger by liquids retention and increased metabolic rate, ultimately some of the main cortisol functions. The CAR may be considered an ancient adaptive feature. Understanding the relationships between brain circuits that modulate emotional memory and cerebral structures that modulate endocrine and autonomic responses to stress may shed light on the processes that regulate resilience and vulnerability when coping with threatening situations.

\section{Conflict of Interest}

The authors declare that they have no competing interests and no financial support to report.

\section{Acknowledgements}

The authors thank Michael Arends for revising and editing the English of the manuscript. 


\section{References}

1. Wust S, Wolf J, Hellhammer DH, et al. The cortisol awakening response - normal values and confounds. Noise and health 2 (2000): 79-88.

2. Pruessner JC, Wolf OT, Hellhammer DH, et al. Free cortisol levels after awakening: a reliable biological marker for the assessment of adrenocortical activity. Life Sci 61 (1997): 2539-2549.

3. McEwen BS. Stress, adaptation, and disease: allostasis and allostatic load. Ann N Y Acad Sci 840 (1998): 33-44.

4. McEwen BS. Physiology and neurobiology of stress and adaptation: central role of the brain. Physiol Rev 87 (2007): 873-904.

5. McEwen BS. Allostasis, allostatic load, and the aging nervous system: role of excitatory amino acids and excitotoxicity. Neurochem Res 25 (2000): 1219-1231.

6. McEwen BS. Interacting mediators of allostasis and allostatic load: towards an understanding of resilience in aging. Metabolism 52 (2003): 10-16.

7. Rutter M. Implications of resilience concepts for scientific understanding. Ann N Y Acad Sci 1094 (2006): $1-12$.

8. Vreeburg SA, Hartman CA, Hoogendijk WJ, et al. Parental history of depression or anxiety and the cortisol awakening response. The British journal of psychiatry : the journal of mental science 197 (2010): 180-185.

9. Karatsoreos IN, McEwen BS. Psychobiological allostasis: resistance, resilience and vulnerability. Trends in cognitive sciences 15 (2011): 576-584.

10. Karatsoreos IN, McEwen BS. Resilience and vulnerability: a neurobiological perspective. F1000Prime Rep 5 (2013): 13.

11. Kozlov AI, Kozlova MA. Cortisol as a marker of stress. Fiziologiia cheloveka 40 (2014):123-136.

12. Kovacs KJ. CRH: the link between hormonal-, metabolic- and behavioral responses to stress. Journal of chemical neuroanatomy 54 (2013): 25-33.

13. Contreras CM, Gutiérrez-García AG. Emotional memory and chemical communication. In: Benitez-King, G, Cisneros-Berlanga C. The Neurobiological Sciences Applied to Psychiatry: From Genes, Proteins, and Neurotransmitters to Behavior. Research Signpost, Kerala 171-188.

14. Gutiérrez-García AG, Contreras CM. Anxiety: an adaptive emotion. In: Durbano, F. New Insights into Anxiety Disorders. INTECH, Rijeka 21-37.

15. LeDoux JE. Emotion circuits in the brain. Annu Rev Neurosci 23 (2000):155-184.

16. Pani L, Porcella A, Gessa GL. The role of stress in the pathophysiology of the dopaminergic system. Molecular psychiatry 5 (2000): 14-21.

17. Vertes RP. Interactions among the medial prefrontal cortex, hippocampus and midline thalamus in emotional and cognitive processing in the rat. Neuroscience 142 (2006): 1-20.

18. Zelikowsky M, Hersman S, Chawla MK, et al. Neuronal ensembles in amygdala, hippocampus, and prefrontal cortex track differential components of contextual fear. The Journal of neuroscience : The official journal of the Society for Neuroscience 34 (2014): 8462-8466.

19. Phillips ML, Drevets WC, Rauch SL, et al. Neurobiology of emotion perception I: The neural basis of normal emotion perception. Biological psychiatry 54 (2003): 504-514. 
20. LeDoux J. The amygdala. Curr Biol 17 (2007): R868-R874.

21. Maren S. Neurotoxic basolateral amygdala lesions impair learning and memory but not the performance of conditional fear in rats. The Journal of neuroscience : The official journal of the Society for Neuroscience 19 (1999): 8696-8703.

22. Nader K, Majidishad P, Amorapanth P, et al. Damage to the lateral and central, but not other, amygdaloid nuclei prevents the acquisition of auditory fear conditioning. Learn Mem 8 (2001): 156-163.

23. Herry C, Ciocchi S, Senn V, et al. Switching on and off fear by distinct neuronal circuits. Nature 454 (2008): 600-606.

24. Sah P, Faber ES, Lopez De Armentia M, et al. The amygdaloid complex: anatomy and physiology. Physiol Rev 83 (2003): 803-834.

25. Pelletier JG, Likhtik E, Filali M, et al. Lasting increases in basolateral amygdala activity after emotional arousal: implications for facilitated consolidation of emotional memories. Learn Mem 12 (2005): 96-102.

26. Villarreal G, King CY. Brain imaging in posttraumatic stress disorder. Seminars in clinical neuropsychiatry 6 (2001): 131-145.

27. Davis M, Whalen PJ. The amygdala: vigilance and emotion. Molecular psychiatry 6 (2001): 13-34.

28. Grace AA, Rosenkranz JA. Regulation of conditioned responses of basolateral amygdala neurons. Physiology and behavior 77 (2002): 489-493.

29. Hilton SM, Zbrozyna AW. Amygdaloid region for defence reactions and its efferent pathway to the brain stem. The Journal of physiology 165 (1963): 160-173.

30. Gunne LM, Reis DJ. Changes in brain catecholamines associated with electrical stimulation of amygdaloid nucleus. Life sciences 11 (1963): 804-809.

31. Romanski LM, LeDoux JE. Equipotentiality of thalamo-amygdala and thalamo-cortico-amygdala circuits in auditory fear conditioning. The Journal of neuroscience: The official journal of the Society for Neuroscience 12 (1992): 4501-4509.

32. Veer IM, Oei NY, Spinhoven P, et al. Endogenous cortisol is associated with functional connectivity between the amygdala and medial prefrontal cortex. Psychoneuroendocrinology 37 (2012): 1039-1047.

33. Iwata J, LeDoux JE, Meeley MP, et al. Intrinsic neurons in the amygdaloid field projected to by the medial geniculate body mediate emotional responses conditioned to acoustic stimuli. Brain Res 383 (1986): 195214.

34. LeDoux JE. Evolution of human emotion: a view through fear. Prog Brain Res 195 (2012): 431-442.

35. Wang Z, Pang RD, Hernandez M, et al. Anxiolytic-like effect of pregabalin on unconditioned fear in the rat: an autoradiographic brain perfusion mapping and functional connectivity study. NeuroImage 59 (2012): 4168-4188.

36. Fanselow MS, Dong HW. Are the dorsal and ventral hippocampus functionally distinct structures? Neuron 65 (2010): 7-19.

37. Bannerman DM, Rawlins JN, McHugh SB, et al. Regional dissociations within the hippocampus--memory and anxiety. Neuroscience and biobehavioral reviews 28 (2004): 273-283.

38. Contreras CM, Molina-Jiménez T, Gutiérrez-García AG. Exposure to an alarm pheromone combined with footshock stress enhances responsivity of the medial amygdala-hippocampus circuit. Am J Psychiatry Neurosci 2 (2014): 83. 
39. van Stegeren AH, Wolf OT, Everaerd W, et al. Endogenous cortisol level interacts with noradrenergic activation in the human amygdala. Neurobiol Learn Mem 87 (2007): 57-66.

40. Carrasco GA, Van de Kar LD. Neuroendocrine pharmacology of stress. Eur J Pharmacol 463 (2003): 235272.

41. Canonaco M, Valenti A, Maggi A. Effects of progesterone on $\left[{ }^{35} \mathrm{~S}\right] \mathrm{t}$-butylbicyclophosphorothionate binding in some forebrain areas of the female rat and its correlation to aggressive behavior. Pharmacology, biochemistry, and behavior 37 (1990): 433-438.

42. Fraile IG, McEwen BS, Pfaff DW. Progesterone inhibition of aggressive behaviors in hamsters. Physiology and behavior 39 (1987): 225-229.

43. Fraile IG, Pfaff DW, McEwen BS. Progestin receptors with and without estrogen induction in male and female hamster brain. Neuroendocrinology 45 (1987): 487-491.

44. Heidbreder CA, Groenewegen HJ. The medial prefrontal cortex in the rat: evidence for a dorso-ventral distinction based upon functional and anatomical characteristics. Neurosci Biobehav Rev 27 (2003): 555579 .

45. Gabbott PL, Warner TA, Jays PR, et al. Prefrontal cortex in the rat: projections to subcortical autonomic, motor, and limbic centers. J Comp Neurol 492 (2005): 145-177.

46. Sierra-Mercado D, Padilla-Coreano N, Quirk GJ. Dissociable roles of prelimbic and infralimbic cortices, ventral hippocampus, and basolateral amygdala in the expression and extinction of conditioned fear. Neuropsychopharmacology: Official publication of the American College of Neuropsychopharmacology 36 (2011): 529-538.

47. Senn V, Wolff SB, Herry C, et al. Long-range connectivity defines behavioral specificity of amygdala neurons. Neuron 81 (2014): 428-437.

48. Risold PY, Swanson LW. Connections of the lateral septal complex. Brain Res Rev 24 (1997): $115-195$.

49. Contreras CM, Rodríguez-Landa JF, Gutiérrez-García AG. The lowest effective dose of fluoxetine in the forced swim test significantly affects the firing rate of lateral septal neurones in the rat. Journal of psychopharmacology (Oxford, England) 15 (2001): 231-236.

50. Yadin E, Thomas E, Grishkat HL, et al. The role of lateral septum in anxiolysis. Physiology and behavior 53 (1993): 1077-1093.

51. Sheehan TP, Chambers RA, Russell DS. Regulation of affect by the lateral septum: implications for neuropsychiatry. Brain research Brain research reviews 46 (2004): 71-117.

52. Lemola S, Perkinson-Gloor N, Hagmann-von Arx P, et al. Morning cortisol secretion in school-age children is related to the sleep pattern of the preceding night. Psychoneuroendocrinology 52 (2015): 297301.

53. Klumpers UM, Veltman DJ, van Tol MJ, et al. Neurophysiological effects of sleep deprivation in healthy adults, a pilot study. PloS one 10 (2015):e0116906.

54. Wright KPJ, Drake AL, Frey DJ, et al. Influence of sleep deprivation and circadian misalignment on cortisol, inflammatory markers, and cytokine balance. Brain Behav Immun 47 (2015): 24-34.

55. Balbo M, Leproult R, Van Cauter E. Impact of sleep and its disturbances on hypothalamo-pituitary-adrenal axis activity. International journal of endocrinology (2010): 759234. 
56. Van Lenten SA, Doane, LD. Examining multiple sleep behaviors and diurnal salivary cortisol and alphaamylase: Within- and between-person associations. Psychoneuroendocrinology 68 (2016): 100-110.

57. Cascio CS, Shinsako J, Dallman MF. The suprachiasmatic nuclei stimulate evening ACTH secretion in the rat. Brain Res 423 (1987): 173-178.

58. Buijs RM, Kalsbeek A, van der Woude TP, et al. Suprachiasmatic nucleus lesion increases corticosterone secretion. Am J Physiol 264 (1993): R1186-R1192.

59. Buijs RM, la Fleur SE, Wortel J, et al. The suprachiasmatic nucleus balances sympathetic and parasympathetic output to peripheral organs through separate preautonomic neurons. J Comp Neurol 464 (2003): 36-48.

60. Kalsbeek A, van der Spek R, Lei J, et al. Circadian rhythms in the hypothalamo-pituitary-adrenal (HPA) axis. Mol Cell Endocrinol 349 (2012): 20-29.

61. Vrang N, Larsen PJ, Mikkelsen JD. Direct projection from the suprachiasmatic nucleus to hypophysiotrophic corticotropin-releasing factor immunoreactive cells in the paraventricular nucleus of the hypothalamus demonstrated by means of Phaseolus vulgaris-leucoagglutinin tract tracing. Brain Res 684 (1995): 61-69.

62. Herman JP, Tasker JG, Ziegler DR, et al. Local circuit regulation of paraventricular nucleus stress integration glutamate GABA-connections. Pharmacol Biochem Behav 71 (2002): 457-468.

63. Moga MM, Moore RY. Organization of neural inputs to the suprachiasmatic nucleus in the rat. J Comp Neurol 389 (1997): 508-534.

64. Csáki A, Kocsis B, Halász B, et al. Localization of glutamatergic/aspartatergic neurons projecting to the hypothalamic paraventricular nucleus studied by retrograde transport of $(3 \mathrm{H}) \mathrm{D}$-aspartate autoradiography. Neuroscience 101 (2000): 637-655.

65. Jacobson L, Akana SF, Cascio CS, et al. Circadian variations in plasma corticosterone permit normal termination of adrenocorticotropin responses to stress. Endocrinology 122 (1988): 1343-1348.

66. Barreto RE, Barbosa-Junior A, Urbinati EC, et al. Cortisol influences the antipredator behavior induced by chemical alarm cues in the Frillfin goby. Hormones and behavior 65 (2014): 394-400.

67. Fischer EK, Harris RM, Hofmann HA, et al. Predator exposure alters stress physiology in guppies across timescales. Hormones and behavior 65 (2014): 165-172.

68. O'Connor CM, Gilmour KM, Van Der Kraak G, et al. Circulating androgens are influenced by parental nest defense in a wild teleost fish. Journal of comparative physiology A, Neuroethology, sensory, neural, and behavioral physiology 197 (2011): 711-715.

69. Sinha AK, Liew HJ, Diricx M, et al. Combined effects of high environmental ammonia, starvation and exercise on hormonal and ion-regulatory response in goldfish (Carassius auratus L.). Aquatic toxicology (Amsterdam, Netherlands) 115 (2012): 153-164.

70. Narayan EJ, Cockrem JF, Hero JM. Sight of a predator induces a corticosterone stress response and generates fear in an amphibian. PloS one 8 (2013): e73564.

71. Zhang JX, Cao C, Gao H, et al. Effects of weasel odor on behavior and physiology of two hamster species. Physiology and behavior 79 (2003): 549-552.

72. Mateo JM. Ecological and hormonal correlates of antipredator behavior in adult Belding's ground squirrels (Spermophilus beldingi). Behavioral ecology and sociobiology 62 (2007): 37-49. 
73. Sheriff MJ, Krebs CJ, Boonstra R. From process to pattern: how fluctuating predation risk impacts the stress axis of snowshoe hares during the 10-year cycle. Oecologia 166 (2011): 593-605.

74. Wang Z, Wang B, Lu J. Behavioral and physiological responses of striped field mice (Apodemus agrarius) to predator odor. Integrative zoology 6 (2011): 334-340.

75. Olsson K, Hydbring-Sandberg E. Exposure to a dog elicits different cardiovascular and behavioral effects in pregnant and lactating goats. Acta veterinaria Scandinavica 53 (2011): 60.

76. Oki C, Atkinson S. Diurnal patterns of cortisol and thyroid hormones in the Harbor seal (Phoca vitulina) during summer and winter seasons. General and comparative endocrinology 136 (2004): 289-297.

77. Sapolsky RM. Stress hormones: good and bad. Neurobiology of disease 7 (2000): 540-542.

78. Sapolsky RM, Romero LM, Munck AU. How do glucocorticoids influence stress responses? Integrating permissive, suppressive, stimulatory, and preparative actions. Endocrine reviews 21 (2000): 55-89.

79. Reser JE: Chronic stress, cortical plasticity and neuroecology. Behav Processes 129 (2016): 105-115.

80. Kalsbeek A, Yi CX, Cailotto C, et al. Mammalian clock output mechanisms. Essays in biochemistry 49 (2011): 137-151.

81. Daly M, Delaney L, Doran PP, et al. The role of awakening cortisol and psychological distress in diurnal variations in affect: a day reconstruction study. Emotion11 (2011): 524-532.

82. Korte SM, Koolhaas JM, Wingfield JC, et al. The Darwinian concept of stress: benefits of allostasis and costs of allostatic load and the trade-offs in health and disease. Neuroscience and biobehavioral reviews 29 (2005): 3-38.

Citation: Carlos M. Contreras, Ana G. Gutiérrez-Garcia. Cortisol Awakening Response: An Ancient Adaptive Feature. Journal of Psychiatry and Psychiatric Disorders 2 (2018): 29-40.

(C) This article is an open access article distributed under the terms and conditions of the 